\title{
MicroRNA-873 inhibits colorectal cancer metastasis by targeting ELK1 and STRN4
}

\author{
Chuannan Fan ${ }^{1, *}$, Biyu Lin ${ }^{1, *}$, Zhengjie Huang ${ }^{3, *}$, Dan Cui, ${ }^{1,}$, Minyi Zhu ${ }^{1}$, Zhenling \\ Ma $^{1}$, Yunda Zhang ${ }^{3}$, Fan Liu ${ }^{2}$ and Yingfu Liu ${ }^{2}$ \\ ${ }^{1}$ State Key Laboratory of Cellular Stress Biology, Innovation Center for Cell Signaling Network, School of Life Sciences, \\ Xiamen University, Fujian Sheng, People's Republic of China \\ ${ }^{2}$ Medical College of Xiamen University, Department of Basic Medical Sciences, Medical College, Xiamen University, Fujian \\ Sheng, People's Republic of China \\ ${ }^{3}$ Department of Surgical Oncology, First Affiliated Hospital of Xiamen University, Fujian Sheng, People's Republic of China \\ *These authors contributed equally to this work
}

Correspondence to: Yingfu Liu, email: liuyingfu@126.com

Fan Liu, email: liufan@xmu.edu.cn

Keywords: colorectal cancer; miR-873; ELK1; STRN4; metastasis

Received: July 03, 2017 Accepted: December 01, 2017 Epub: January 02, 2018 Published: June 25, 2019

Copyright: Fan et al. This is an open-access article distributed under the terms of the Creative Commons Attribution License 3.0 (CC BY 3.0), which permits unrestricted use, distribution, and reproduction in any medium, provided the original author and source are credited.

\section{ABSTRACT}

MicroRNAs (miRNAs) are a group of small non-coding RNAs that directly bind to the 3'-untranslated-region (3'UTR) of mRNA, thereby blocking gene expression post-transcriptionally. Accumulating evidence prove that microRNA-873 (miR-873) functions as a promoter or suppressor in various cancers, while whether it affects the progression of colorectal cancer (CRC) is yet unknown. Here we found that miR-873 was downregulated in human CRC clinical samples, mouse CRC specimens and cell lines with high metastatic potential. We also demonstrated that low miR873 expression was closely associated with poor prognosis of CRC. Overexpressing miR-873 suppressed proliferation and metastasis of CRC cells both in vitro and in vivo, while inhibiting miR-873 expression promoted the proliferation, migration and invasion in vitro. Moreover, miR-873 exerted its function by perturbing the ERK-CyclinD1 pathway and the epithelial-mesenchymal transition (EMT) process. Furthermore, we revealed that miR-873 acted as a tumor-suppressive microRNA by directly binding to the 3'UTRs of ELK1 and STRN4 and suppressed their expression. Our study uncovered an inhibitory role of miR-873 in CRC progression and might provide a promising marker for CRC diagnosis and prognosis.

\section{INTRODUCTION}

Colorectal cancer $(\mathrm{CRC})$ is one of the most common malignancies. It is the fourth leading cause of cancer mortalities in men and the third one of cancer mortalities in women [1]. The death rate has decreased by approximately $3 \%$ every year during the past decades. However, the metastatic lesion formed by primary tumor is still a major cause of CRC death [2]. Although increasing evidence has uncovered diverse regulatory mechanisms involved in CRC progression, pivotal drivers and inhibitors related to this process are largely unknown.
Genomic studies shows that misregulation of TGF- $\beta$, WNT and EGFR signaling pathways are general events in CRC progression [3-6]. CRC with chromosomal instability (CIN) and microsatellite instability (MSI) is more inclined to acquire hypermethylation of key genes, which leads to gain higher invasive ability and eventually form macrometastases [7, 8]. MiRNAs are a large class of noncoding RNAs that participate in the regulation of mRNA translation or/and stability by directly binding to target mRNAs. Dysregulation of miRNAs results from genetic or epigenetic modifications is corroborated to be common in the invasion-metastasis cascade of CRC $[9,10]$. 
MiR-873, a miRNA located on the chromosome 9, has been identified as a tumor suppressor in glioblastoma [11], ovarian cancer [12] and breast cancer [13]. However, it has been proved to be an oncogene in lung adenocarcinoma [14]. Of note, whether miR-873 affects $\mathrm{CRC}$ progression is yet unclear. Interestingly, a previous study proves that the promoter of miR-873 was widely hypermethylated in most CRC cell lines, which implies that miR-873 may be a potential tumor suppressor in CRC [15]. In this study, we aimed to interrogate the expression characteristics of miR-873 and elucidate the functional importance of it by in vitro and in vivo methods. In our study, miR-873 was downregulated in CRC patient samples and two mouse CRC models. Furthermore, miR-873 level was inversely correlated to the metastatic potential of clinical specimens and CRC cell lines. Overexpressing miR-873 inhibited CRC cell proliferation, migration and invasion in vitro, while inhibition of miR873 increased CRC cell proliferation, migration and invasion. MiR-873 exerted its tumor-suppressive role by inhibiting the ERK-CyclinD1 axis and the EMT process. We further demonstrated that miR-873 suppressed CRC cell growth and liver metastasis in vivo. Very interestingly, we found that ELK1 and STRN4 were direct targets of miR-873. In conclusion, miR-873 functions as a vital molecular participant in CRC growth and metastasis and our data may shed light on further use of miR-873 as a new diagnostic and prognostic biomarker of CRC.

\section{RESULTS}

\section{MiR-873 may act as a tumor suppressor in CRC}

To determine the clinical significance of miR-873 expression, we first detected the expression levels of miR873 in paired human clinical samples of CRC. MiR-873 expression was downregulated in 43 of 55 (78.2\%) CRC tumor specimens compared to their normal counterparts (Figure 1A). The miR-873 levels in CRC tumors (median $=0.60)$ were significantly lower $(P<0.001)$ than that in normal colon samples (median $=2.22$ ). Moreover, in primary tumor samples of patients with liver metastases, miR-873 expression was even lower than those without liver metastases (Figure 1B). And, the relationships of miR-873 expression with clinicopathological factors of CRC was shown in Table 1. The decrease of miR-873 expression was found to be significantly related to distant metastasis. However, no significant correlations were found between miR-873 expression and other factors including age, gender, clinical stage and lymph node metastasis. Interestingly, miR-873 levels in CRC cell lines with high metastatic potential (SW620, HCT116 and LoVo) were significantly lower than those cell lines with low metastatic potential (HCT8, SW480, LS174T, HT29 and RKO) and normal colon epithelial cell line NCM460
(Figure 1C). The AOM/DSS mouse model is a colitisassociated CRC model and the $A P C^{\mathrm{Min}+}$ mouse model is a spontaneous CRC model. These two models can mimic most of the cases in human CRC progression [16-18]. We interrogated miR-873 expression in samples from these two kinds of mouse models. As shown in Figure 1D, miR-873 expression in tumor tissues from the AOM/ DSS-administrated group was significantly lower than that in normal colon tissues from control group. Likewise, miR-873 expression was decreased in tumor tissues from $A P C^{\mathrm{Min}^{+}}$mice compared with normal colon tissues from wild type mice (Figure 1E). These data indicated that miR-873 may be a tumor suppressor and is negatively correlated with the metastatic potential of CRC.

\section{Overexpression of miR-873 inhibits CRC cell proliferation, migration and invasion in vitro}

To reveal the biological roles of miR-873 in CRC, we overexpressed pre-miR-873 in two CRC cell lines LoVo and HCT116 with high metastatic potential (Figure 2A). MTT assay was performed to examine the effect of miR-873 overexpression on CRC cell proliferation. As shown in Figure 2B, ectopic expression of miR-873 significantly inhibited proliferation of LoVo and HCT116 cells. Colony formation assays showed that miR-873 overexpressing cells formed less colonies than control cells (Figure 2C), which also indicated that miR-873 inhibits CRC cell proliferation in vitro. We further found that the phosphorylated level of ERK $1 / 2$ and the protein level of CyclinD1 were decreased upon overexpression of miR-873 in LoVo and HCT116 cells (Figure 2D). Therefore, we speculated that miR-873 may exert its tumor-suppressive role by affecting the ERK-CyclinD1 axis which is one of the most essential pathways that control cell proliferation [19]. Since miR-873 expression was shown to be negatively correlated with the metastatic ability in CRC clinical samples and cell lines, we further performed Transwell assays to examine whether miR873 could inhibit migration and invasion of CRC cells. As a result, overexpression of miR-873 in LoVo and HCT116 cells dramatically suppressed their migration (Figure 2E) and invasion (Figure 2F). We further wanted to know how miR-873 elicits its metastasisinhibitory function. EMT is a prominent process for tumor cells to dedifferentiate from an epithelial into a more mesenchymal phenotype, upon which they become more migratory and invasive and gain metastatic ability [20]. Therefore, we analyzed the expressions of epithelial markers E-cadherin and $\alpha$-E-catenin as well as mesenchymal markers $\mathrm{N}$-cadherin and Vimentin. Interestingly, ectopic expression of miR-873 made the expression of epithelial markers upregulated while expression of mesenchymal markers downregulated (Figure 2G). Altogether, these results indicated that miR- 
Table 1: Relationships between miR-873 expression levels with clinicopathological factors in CRC

\begin{tabular}{|c|c|c|c|c|}
\hline \multirow[t]{2}{*}{ Variables } & \multirow{2}{*}{$N$} & \multicolumn{2}{|c|}{ miR-873 } & \multirow{2}{*}{$P$} \\
\hline & & Low & High & \\
\hline Age (years) & & & & 0.200 \\
\hline$>61$ & 29 & 21 & 8 & \\
\hline$\leq 61$ & 26 & 24 & 2 & \\
\hline Gender & & & & 0.230 \\
\hline Male & 35 & 27 & 8 & \\
\hline Female & 20 & 18 & 2 & \\
\hline Clinical stage & & & & 0.190 \\
\hline I-II & 16 & 12 & 4 & \\
\hline III-IV & 39 & 33 & 6 & \\
\hline pN stage & & & & 0.057 \\
\hline No & 14 & 9 & 5 & \\
\hline N1-N2 & 41 & 36 & 5 & \\
\hline Distant metastasis & & & & $0.031^{*}$ \\
\hline No & 42 & 33 & 9 & \\
\hline Yes & 13 & 12 & 1 & \\
\hline
\end{tabular}

${ }^{*} P<0.05$ by Student's $t$-test

873 could inhibit CRC cell proliferation, migration and invasion in vitro significantly.

\section{Inhibition of miR-873 promotes $\mathrm{CRC}$ cell proliferation, migration and invasion in vitro}

Because miR-873 overexpression suppressed CRC cell proliferation, migration and invasion, we wondered if inhibition of miR-873 can augment CRC cell proliferation and motility. We applied a miR-873 inhibitor to block endogenous miR-873 expression in HCT8 cells (Figure $3 \mathrm{~A}$ ) whose metastatic ability is relative lower and its miR873 level is the highest among all the CRC cell lines we used (Figure 1C). When miR-873 level was decreased, cell proliferation rate was significantly increased as detected by MTT assay (Figure 3B) and colony formation assay (Figure 3C). Moreover, the phosphorylated level of ERK $1 / 2$ and the protein level of CyclinD1 were increased after miR-873 inhibition (Figure 3D). We subsequently explored whether miR-873 knockdown could increase the proliferation, migration and invasion of HCT8 cells. As shown in Figure 3E, cell migration and invasion were dramatically strengthened upon miR-873 inhibition. Moreover, protein levels of E-cadherin and $\alpha$-E-catenin were downregulated and the levels of $\mathrm{N}$-cadherin and Vimentin were upregulated in HCT8 cell transfected with miR-873 inhibitor (Figure 3F). Collectively, our loss-offunction data indicated that inhibition of miR-873 result in the promotion of CRC cell proliferation, migration and invasion.

\section{Overexpressing miR-873 suppresses $\mathrm{CRC}$ cell growth and liver metastasis in vivo}

The above results revealed that miR-873 overexpression could inhibit cell proliferation, migration and invasion in vitro, which prompted us to test whether it can affect these traits in vivo. We first infected LoVo and HCT116 cells with a lentivirus that could stably overexpress a Luciferase gene, followed by infecting these two Luciferase-labeled cells with lentiviruses encoding the vector or pre-miR-873. Then, stable infected LoVo and HCT116 cells were subcutaneously injected into nude mice and bioluminescence imaging was performed after 4 weeks. As shown in Figure 4A, LoVo cells with miR-873 overexpression formed smaller tumors compared with control cells. We then isolated the xenograft tumors and found the weight of LoVomiR-873 tumors was significantly decreased compared with LoVo-Control tumors (Figure 4A). Similarly, we observed ectopic expression of miR-873 in HCT16 cells also dramatically suppresses tumor growth in vivo (Figure 4B). And then, the expression of proliferation marker Ki67 in the isolated tumors was further detected. The proportion of Ki67-positive cells in tumors formed by miR-873 overexpressing cells were much lower than that in tumors formed by control cells (Figure 4C). Liver is the most vital target organ for metastatic CRC and liver metastasis is the direct cause of CRC death [21]. Thus, we further assessed the metastatic ability of miR-873overexpressing cells by injecting them into nude mice 
intrasplenically to construct an experimentally metastatic model. Bioluminescence imaging results showed that LoVo (Figure 4D) and HCT116 (Figure 4E) cells with miR-873 overexpression formed less hepatic metastatic nodules which were validated by H\&E staining of liver slices (Figure 4F). In summary, these above results indicated that miR-873 could inhibit CRC cell growth and metastasis in vivo.

\section{ELK1 and STRN4 are direct targets of miR-873}

MiRNAs exert their biological roles by targeting the 3'UTR of mRNAs, resulting in mRNA degradation and/ or translational inhibition. On the basis of above results which implied miR-873 may serve as a tumor-suppressive miRNA, we further aimed to identify its targets. Therefore, we applied the most common in silico prediction algorithm TargetScan to mine the candidate targets of miR-873.

\section{A}

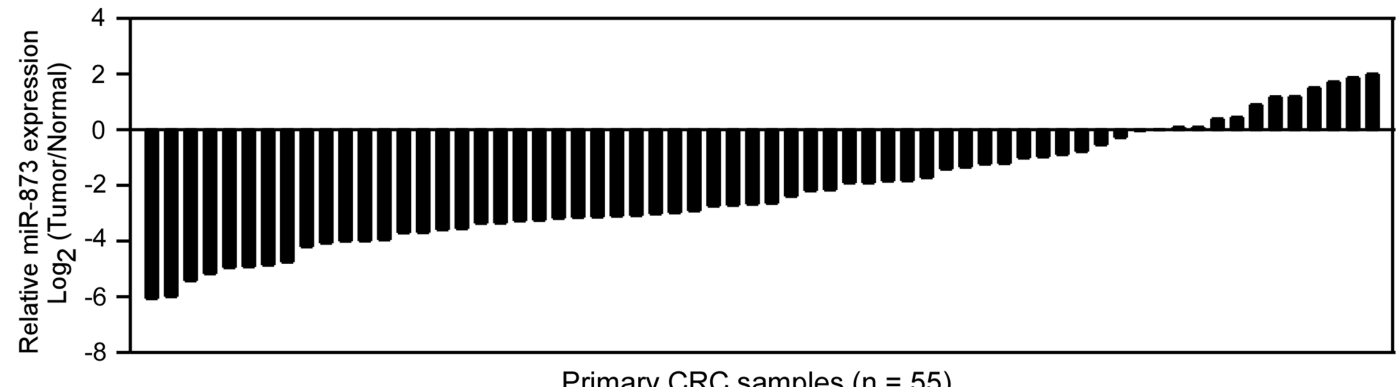

$\mathrm{B}$

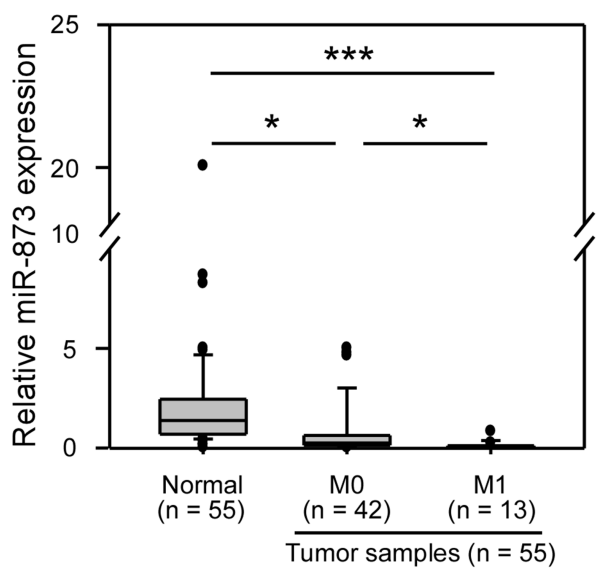

$\mathrm{D}$

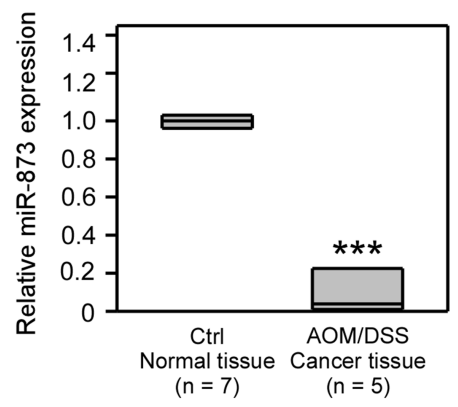

C

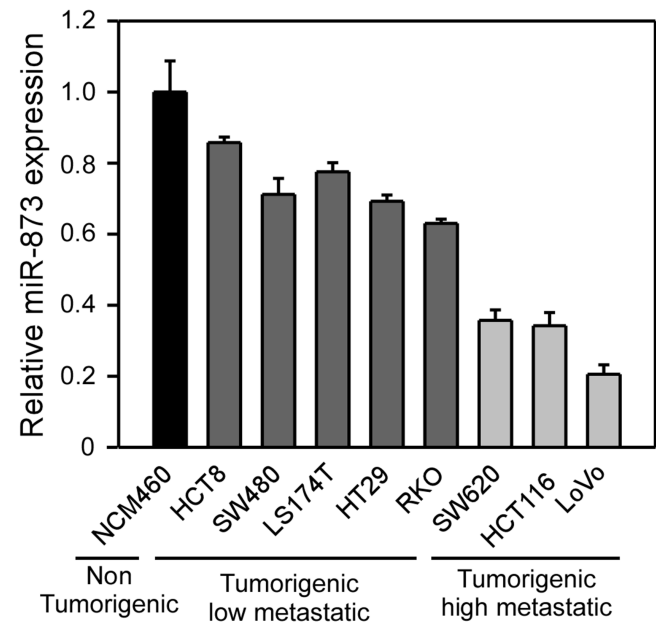

$E$

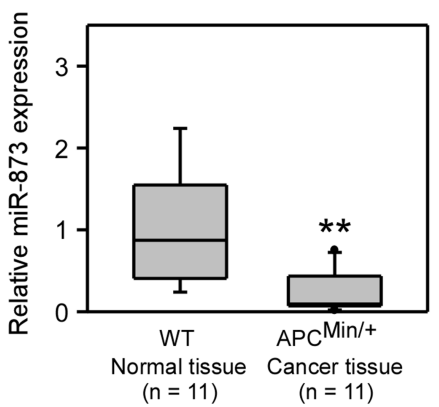

Figure 1: MiR-873 was downregulated in CRC clinical samples, mouse models and CRC cell lines. (A) qRT-PCR analysis of miR-873 levels in 55 paired CRC clinical specimens. (B) Corelation between miR-873 levels and the distant metastasis status of CRC samples. (C) qRT-PCR analysis of miR-873 levels in normal colon cell line and CRC cell lines with different metastatic potential. (D, E) qRT-PCR analysis of miR-873 expression in AOM/DSS mouse model (D) and $A P C^{\text {Min }}{ }^{+}$mouse model (E). Data (mean \pm SEM) are representative of three technique replicates. ${ }^{*} P<0.05 ;{ }^{* *} P<0.01 ;{ }^{* * *} P<0.001$. 
And we selected seven oncogenes that have been proved to affect cell proliferation or/and mobility. The mRNA levels of CDK6, ELK1, MyoB1, STRN4, TRAF2 and WASF2 were revealed to be significantly decreased after ectopic expression of miR-873 in both LoVo and HCT116 cells (Figure 5A and 5B). Moreover, we performed Dual Luciferase Reporter Assay to verify whether there were direct interactions between miR-873 and these genes. MiR-873 and the 3'UTR elements of these genes were co-transfected into $293 \mathrm{~T}$ cells. As showed in Figure 5C,
A

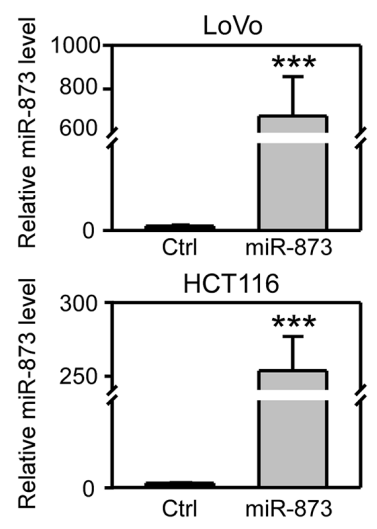

C
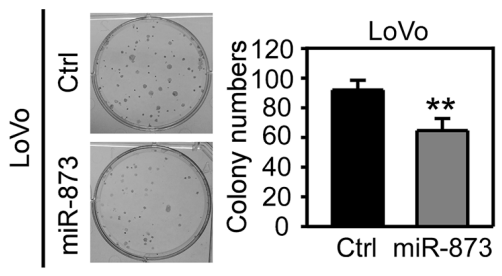

B
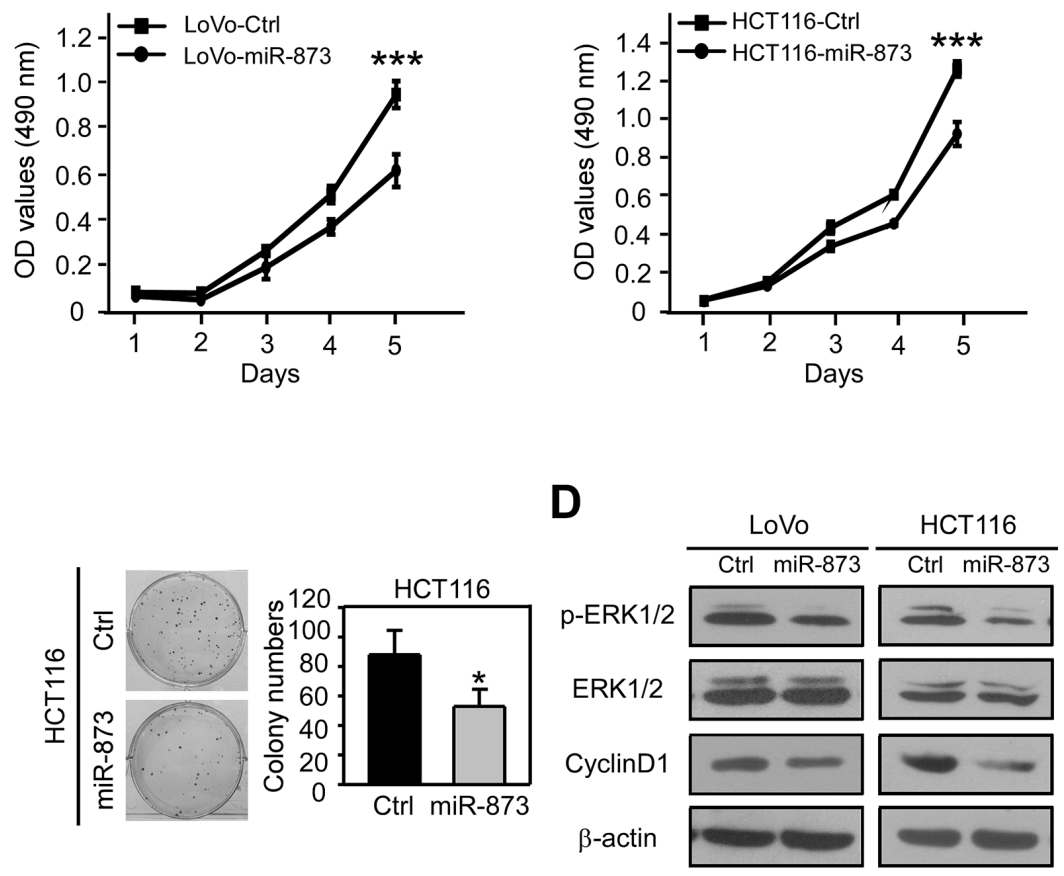
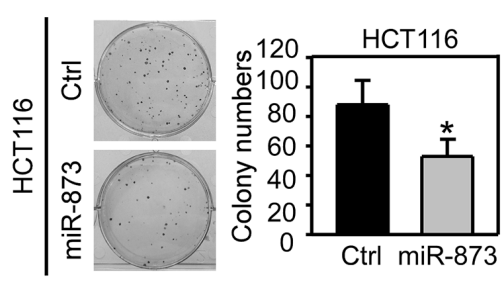

D

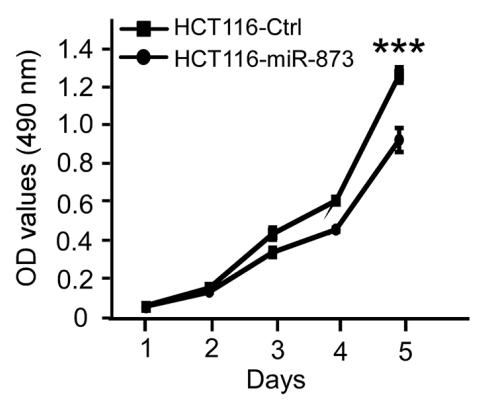

G
E
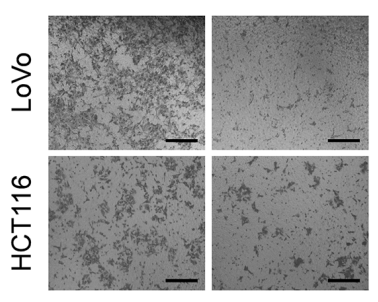

Ctrl

$\mathrm{F}$

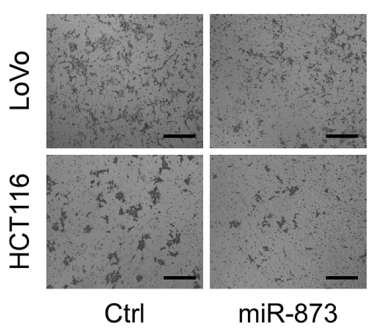

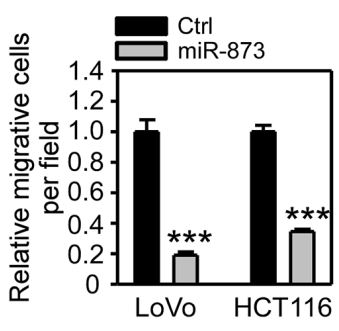
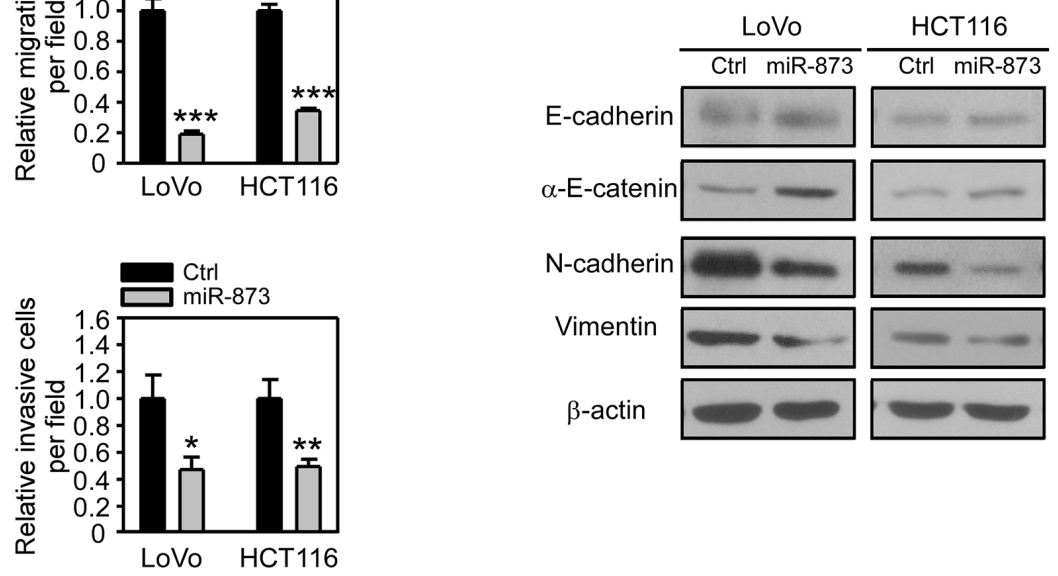

Figure 2: MiR-873 inhibits CRC cell proliferation, migration and invasion in vitro. (A) qRT-PCR analysis of miR-873 levels in LoVo and HCT116 stable infected cell lines. (B) MTT assay of LoVo-control and LoVo-miR-873 cells (left) and HCT116-control and HCT116-miR-873 cells (right). (C) Representative images and quantification of colonies formed by LoVo-control and LoVo-miR-873 cells (left) and HCT116-control and HCT116-miR-873 cells (right). (D) Western blotting analysis of p-ERK1/2, ERK1/2 and CyclinD1 in LoVo/ HCT116-control and LoVo/HCT116-miR-873 cells. $\beta$-actin was used as a loading control. (E, F) Representative images and quantification of cell migration (E) and invasion (F) assays of LoVo/HCT116-control and LoVo/HCT116-miR-873 cells. (G) Western blotting analysis of EMT-related markers in LoVo/HCT116-control and LoVo/HCT116-miR-873 cells. $\beta$-actin was used as a loading control. Data (mean \pm SEM) are representative of 3 technique replicates. Scale bars, $100 \mu \mathrm{m} .{ }^{*} P<0.05 ;{ }^{* *} P<0.01 ;{ }^{* * *} P<0.001$. 
overexpressing miR-873 led to the decrease of Luciferase activity of the 3'UTRs of ELK1 and STRN4 rather than 3'UTRs of CDK6, MyoB1, TRAF2 and WASF2. However, suppression of Luciferase activity was abolished when the putative binding sequences of miR-873 were mutated (Figure 5D and 5E). The mRNA levels of ELK1 and STRN4 in HCT8 cells transfected with miR-873 inhibitor was further detected. Upon miR-873 inhibition, ELK1 and STRN4 showed higher mRNA levels (Figure 5F). Consistent with the qRT-PCR results, the protein levels of ELK1 and STRN4 were decreased in LoVo and HCT116 cells overexpressing miR-873 while their expression was increased in HCT8 cells transfected with the miR-873 inhibitor (Figure 5G). These data demonstrated that miR873 could block ELK1 and STRN4 expression in CRC cells by binding to their 3'UTRs.

\section{Restoration of ELK1 and STRN4 can partially rescue phenotypes restrained by $\mathrm{miR}-873$}

To further estimate whether ELK1 and STRN4 are vital downstream targets of miR-873, we analyzed the proliferation and migration of CRC cells through restoration of ELK1 and STRN4 expression. To this end, miR-873-overexpressing HCT116 cells were transfected with plasmids encoding ELK1 and STRN4 which lack 3'UTR elements, and their protein levels were recovered (Figure 6A). After restoring ELK1 and STRN4, MTT and colony formation assays showed that the inhibitory effects of miR-873 on HCT116 cell proliferation were obviously alleviated (Figure 6B and 6C). Moreover, reactivation of ELK1 and STRN4 in HCT116 cells partially reversed the suppression of migratory ability due to miR873 overexpression (Figure 6D). Taken together, we
A

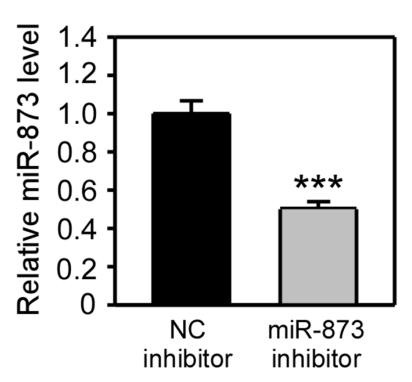

B

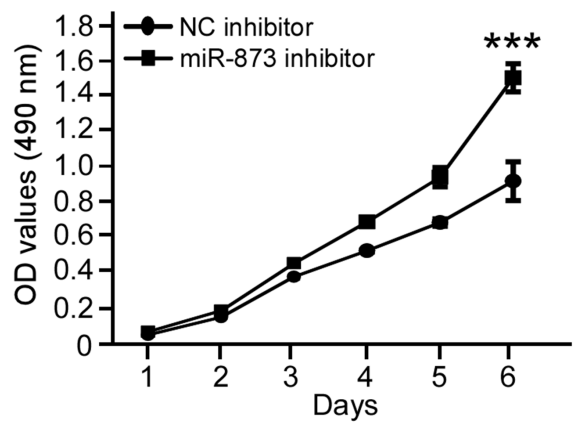

C
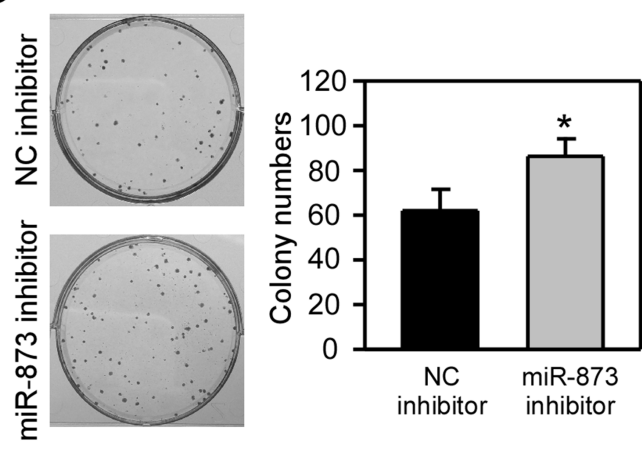

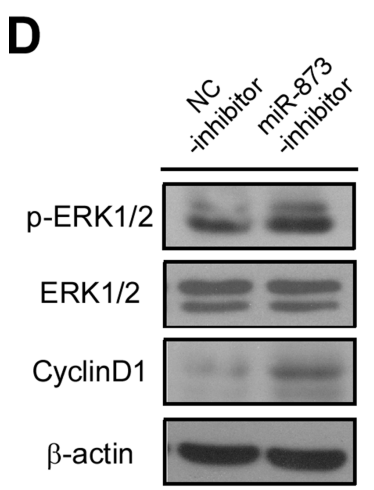

E

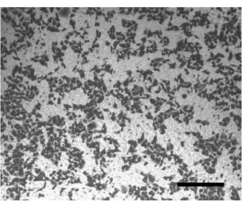

NC inhibitor

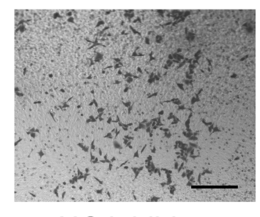

NC inhibitor

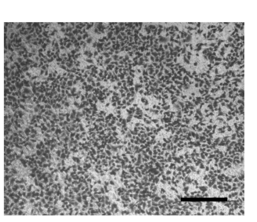

miR-873 inhibitor

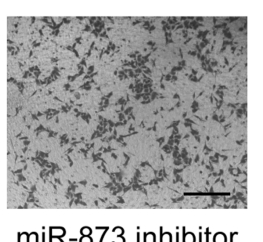

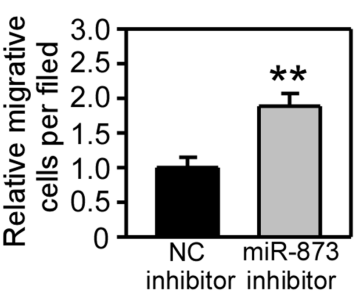

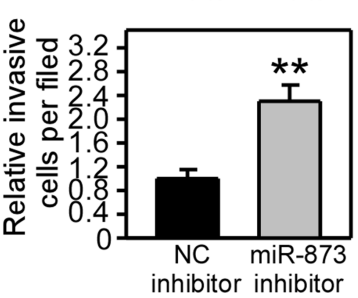

$\mathbf{F}$

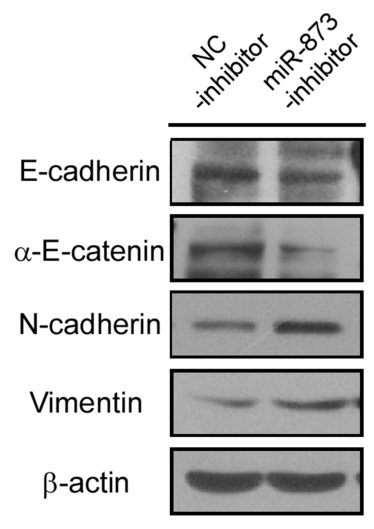

Figure 3: MiR-873 inhibition promotes CRC cell proliferation, migration and invasion in vitro. (A) $\mathrm{qRT}-\mathrm{PCR}$ analysis of miR-873 levels in HCT8 cells transfected with negative control inhibtor (HCT8-NC inhibitor) and miR-873 inbihitor (HCT8-miR-873 inhibitor). (B) MTT assay of HCT8-NC inhibitor and HCT8-miR-873 inhibitor cells. (C) Representative images and quantification of colonies formed by HCT8-NC inhibitor and HCT8-miR-873 inhibitor cells. (D) Western blotting analysis of p-ERK1/2, ERK1/2 and CyclinD1 in HCT8-NC inhibitor and HCT8-miR-873 inhibitor cells. (E) Representative images and quantification of cell migration and invasion assays of HCT8-NC inhibitor and HCT8-miR-873 inhibitor cells. (F) Western blotting analysis of EMT-related markers in HCT8NC inhibitor and HCT8-miR-873 inhibitor cells. Data (mean \pm SEM) are representative of 3 technique replicates. Scale bars, $100 \mu \mathrm{m} .{ }^{*} P$ $<0.05 ;{ }^{* *} P<0.01 ;{ }^{* * *} P<0.001$. 


\section{A}
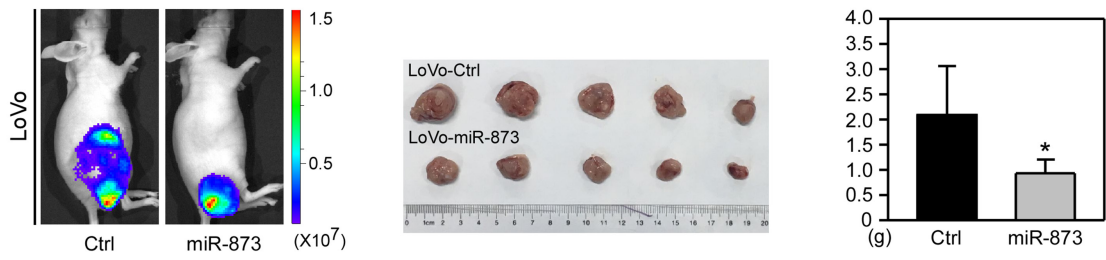

B
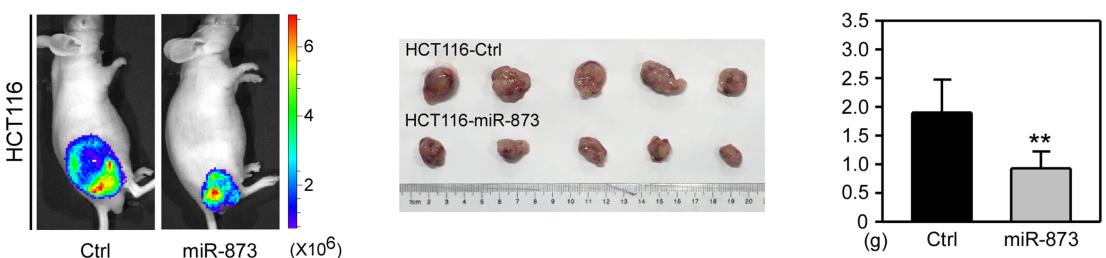

C
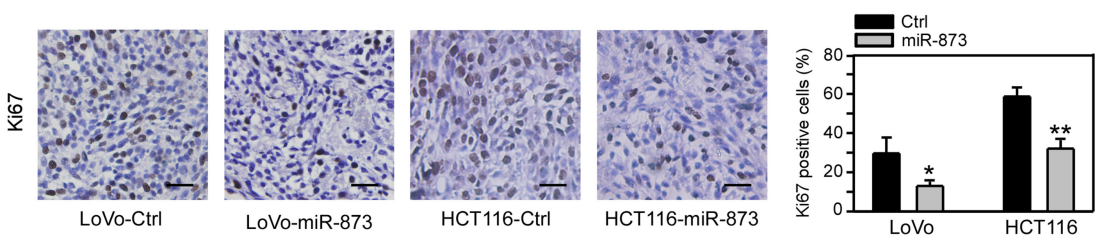

D
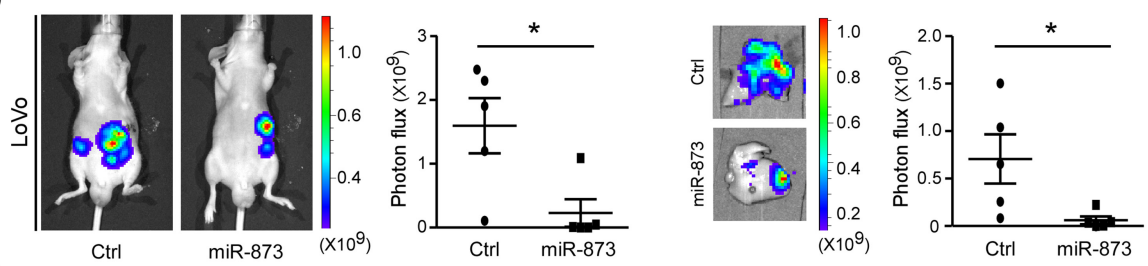

$\mathbf{E}$

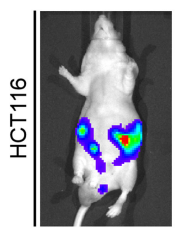

Ctrl
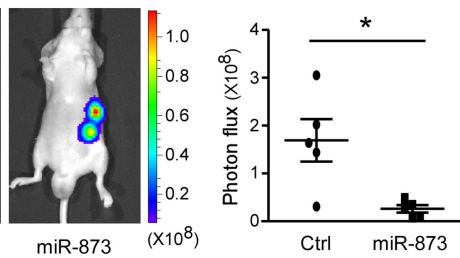
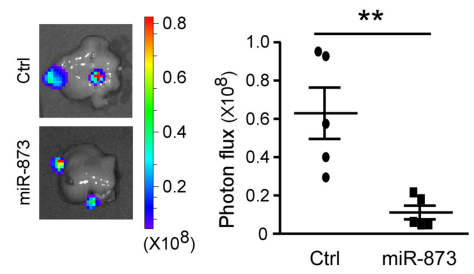

$\mathbf{F}$
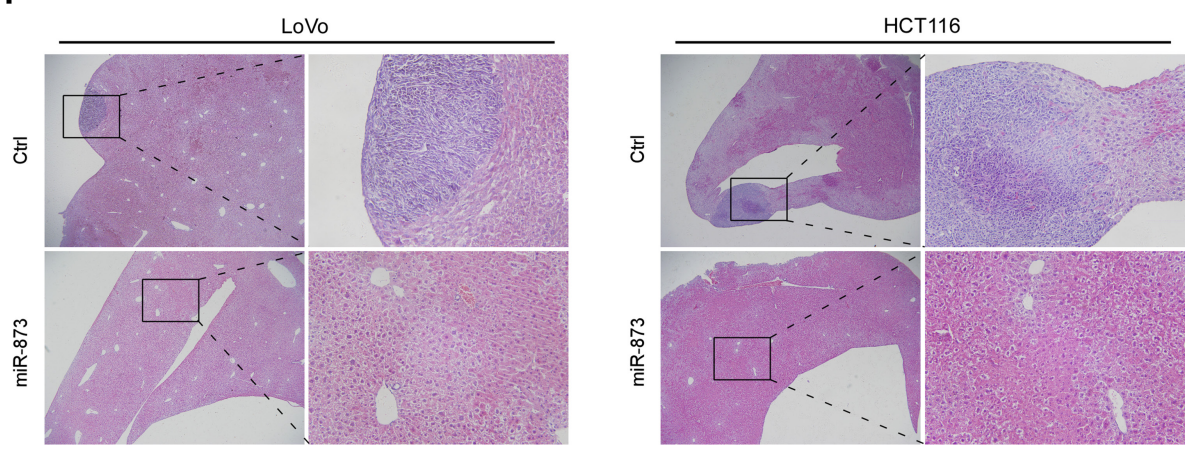

Figure 4: MiR-873 suppresses CRC cell growth and liver metastasis in vivo. (A) Images of bioluminescence of mice that was subcutaneously injected with LoVo-control and LoVo-miR-873 cells (left). Image (middle) and weight quantification (right) of subcutaneous tumors formed by LoVo-control and LoVo-miR-873 cells. (B) Images of bioluminescence of mice that was subcutaneously injected with HCT116-control and HCT116-miR-873 cells (left). Image (middle) and weight quantification (right) of subcutaneous tumors formed by HCT116-control and HCT116-miR-873 cells. (C) Representative images of IHC staining and quantification of Ki67-positve cells rate in tumors formed by LoVo/HCT116-control and LoVo/HCT116-miR-873 cells. (D) Images and quantification of bioluminescence imaging of mice (left) and livers (right) intrasplenically injected with LoVo-control and LoVo-miR-873 cells. (E) Images and quantification of bioluminescence imaging of mice (left) and livers (right) intrasplenically injected with HCT116-control and HCT116-miR-873 cells. Scale bars, $100 \mu \mathrm{m}$. (F) H\&E of livers intrasplenically injected with LoVo-control and LoVo-miR-873 cells or HCT116-control and HCT116-miR-873 cells. Original magnification, $\times 40$ (left of each cell), $\times 200$ (close-up, right of each cell) ${ }^{*} P<0.05 ;{ }^{* *} P<0.01$. 
indicated that miR-873 decreases the proliferation and motility of CRC cells at least partially by targeting ELK1 and STRN4.

\section{DISCUSSION}

MiRNAs are a large class of non-coding RNAs which have been well proved to function as modulators in tumorigenesis and cancer progression [22, 23]. As a very

A

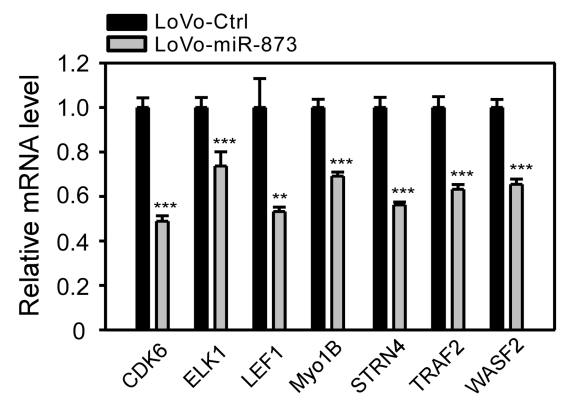

common malignancy, CRC was reported to be widely regulated by miRNAs. MiR-1269a, a miRNA activated by TGF- $\beta$, promotes TGF- $\beta$ signaling via targeting Smad7 and HOXD10, thereby forming a positive feedback loop and increases invasion and metastasis of CRC cells [24]. Recently, we revealed that miR-543 acts as a onco-suppressor miRNA by restraining the RAS-MEKERK-CyclinD1 axis for growth inhibition and targeting HMGA2 and MTA1 for mobility suppression [9]. To date,

B

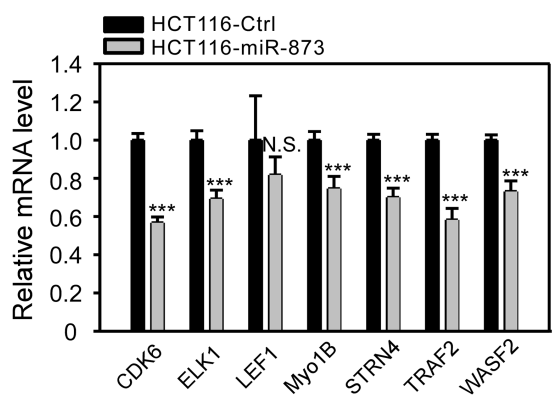

C

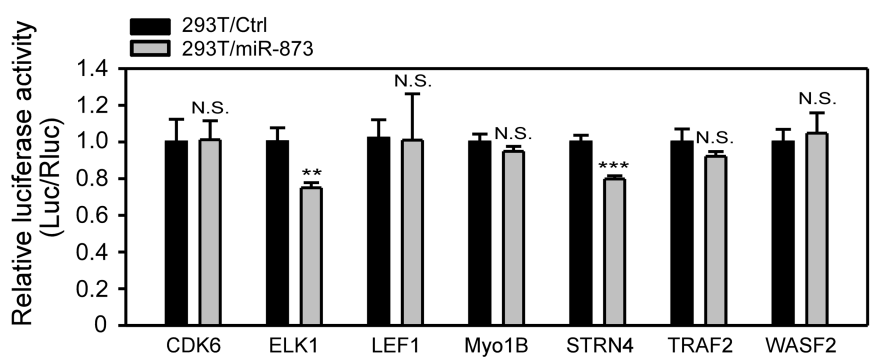

D

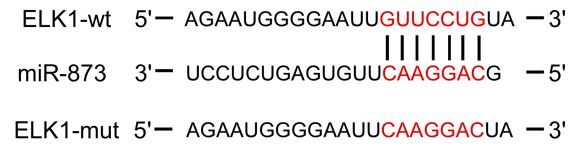

STRN4-wt 5'- GGUCCCCUUCCAGGUUCCUGUU - 3' miR-873 3 - UCCUCUGAGUGUUCAAGGACG $-5^{\prime}$ STRN4-mut 5'- GGUCCCCUUCCAGCAAGGACUU - 3'

$\mathbf{F}$

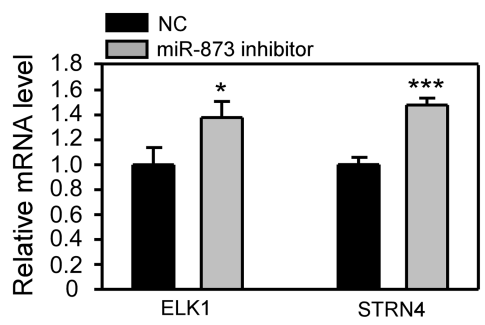

E

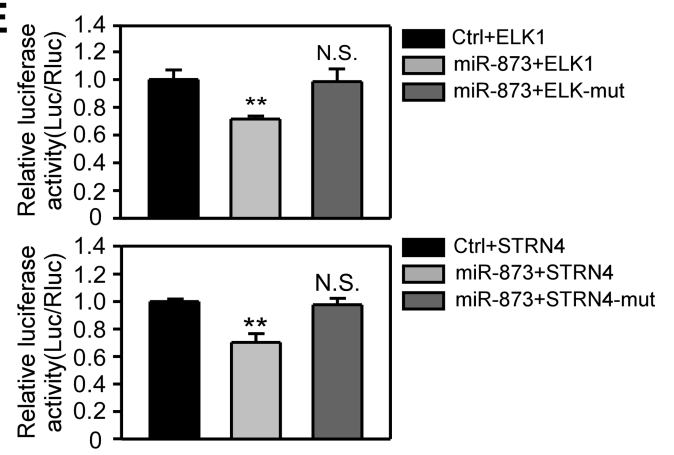

G

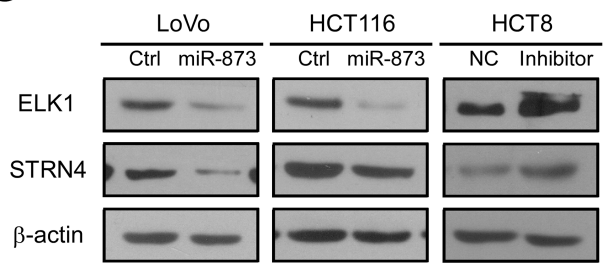

Figure 5: ELK1 and STRN4 are direct targets of miR-873. (A, B) qRT-PCR analysis of mRNA levels of candidate genes in LoVo-control and LoVo-miR-873 cells (A) and HCT116-control and HCT116-miR-873 cells (B). (C) Dual Luciferase reporter assays of 3'UTRs of candidate genes upon transfection of miR-873 in 293T cells. (D) Schematic of mutations generetated in miR-873 binding regions in 3'UTRs of ELK1 and STRN4. (E) Dual Luciferase reporter assays of wild-type or mutant 3'UTRs of candidate genes upon transfection of miR-873 in 293T cells. (F) qRT-PCR analysis of ELK1 and STRN4 mRNA levels in HCT8-NC inhibitor and HCT8-miR-873 inhibitor cells. (G) Western blotting analysis of ELK1 and STRN4 protein levels in LoVo/HCT116-control, LoVo/HCT116-miR-873 cells, HCT8NC inhibitor and HCT8-miR-873 inhibitor cells. ${ }^{*} P<0.05 ;{ }^{* *} P<0.01 ;{ }^{* * *} P<0.001$; N.S. no significance. 
however, roles of miR-873 in CRC are yet unknown. In the present study, we found that miR-873 level was significantly decreased in CRC patient specimens, especially in samples with metastatic potential. Notably, there was a negative correlation between miR-873 expression and the metastatic ability of CRC cell lines. Gain-of-function experiments revealed that proliferation, migration and invasion of CRC cells were suppressed

A

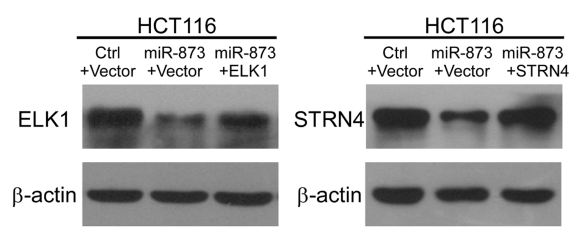

C

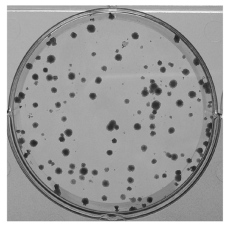

Ctrl+Vector

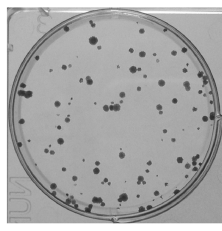

miR-873+ELK1

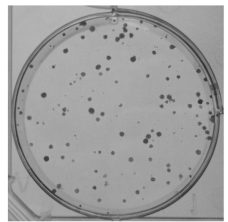

miR-873+Vector

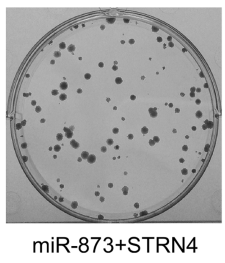

after ectopic expression of miR-873 in vitro and in vivo. In contrast, miR-873 depletion dramatically promoted CRC cell growth and motility in vitro. Furthermore, miR873 overexpression decreased the phosphorylation of ERK1/2 and the level of CyclinD1, which may elicit the proliferation blockage. The EMT process, a prominent driver for tumor metastasis, was also retardant upon overexpression of miR-873. To find the direct targets

B
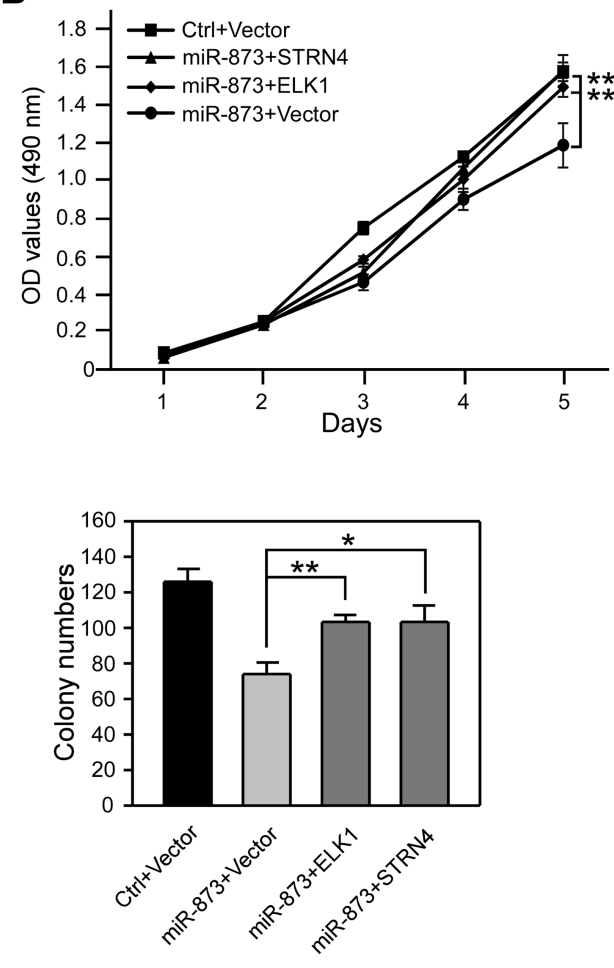

\section{D}

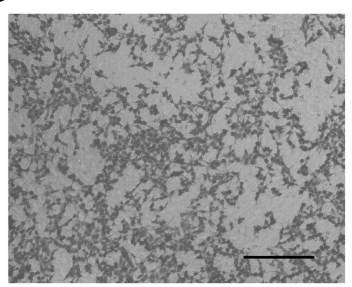

HCT116-Ctrl+Vector

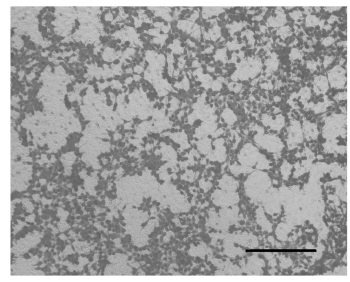

HCT116-miR-873+ELK1

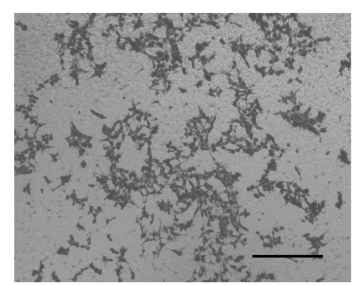

HCT116-miR-873+Vector

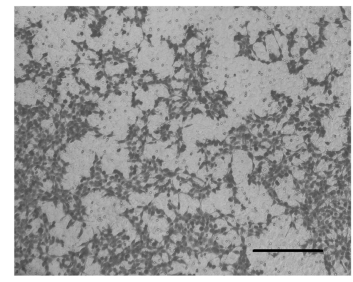

HCT116-miR-873+STRN4

Figure 6: Re-activation of ELK1 and STRN4 can partially rescue phenotypes restrained by miR-873. (A) Western blotting analysis of ELK1 and STRN4 protein levels in HCT116-control and HCT116-miR-873 cells re-expressing ELK1 or STRN4. (B) MTT assays of HCT116-control and HCT116-miR-873 cells re-expressing ELK1 or STRN4. (C) Representative images and quantification of colonies formed by HCT116-control and HCT116-miR-873 cells re-expressing ELK1 or STRN4. (D) Representative images and quantification of cell migration and invasion assays of HCT116-control and HCT116-miR-873 cells re-expressing ELK1 or STRN4. Data $($ mean $\pm \mathrm{SEM})$ are representative of 3 technique replicates. Scale bars, $100 \mu \mathrm{m} .{ }^{*} P<0.05 ;{ }^{* *} P<0.01$. 
of miR-873, we combined an in silico prediction tool with functional validation experiments. Ultimately, we identified ELK1 and STRN4 as downstream target genes for miR-873 to exert its tumor-suppressive roles.

MiR-873 has been demonstrated to play diverse roles in various types of cancers. It is first identified to suppress breast cancer cell proliferation and enhance tamoxifen resistance by targeting CDK3 [13]. In glioma cells, miR-873 overexpression results in the decrease of Bcl-2, followed by suppressing cell proliferation, motility and promoting apoptosis in cells which are cisplatin resistant [25]. Similarly, miR-873 acts as an apoptosis sensitizer of ovarian cells by targeting ABCB1 [12]. Futhermore, IGF2BP1 has been proved to be a target of miR-873 in glioblastoma cells and their growth and metastasis are inhibited by miR-873 [11]. However, miR-873 has been reported as an oncogene in lung adenocarcinoma by inhibiting its downstream target SRCIN1 [14]. The multiple functions of miR-873 in different cancer types may depend on the specific cellular contexts. Previous study indicated the hypermethylation of miR-873 promoter is a common event in CRC cell lines. Consistently, our qRT-PCR results revealed miR-873 was downregulated in CRC patient samples. It is worth noting that in addition to hypermethylation, other regulatory mechanisms involved in the dysregulation of miR-873 in $\mathrm{CRC}$ need further investigation.

ELK1 is a crucial transcription factor that mediates the MEK-ERK signaling transduction. It functions as an activator for early oncogene expression like c-Fos when tumor cells sense various extracellular signals and promotes cell proliferation [26, 27]. Moreover, ELK1 plays a key role in eliciting EMT in non-small cell lung cancer (NSCLC) and gastric cancer cells [28, 29]. STRN4 is a member of the striatin family which forms a large complex with proteins of the germinal center kinase family [30]. It is highly expressed in a variety of cancer cells including HCT116 and knockdown of STRN4 dramatically decreases proliferation and migration of HCT116 cells [31]. Our data indicated that miR-873 directly binds and inhibits ELK1 and STRN4, thereby suppressing growth and metastasis of CRC cells. Given the multi-targeting trait of miRNA, there may be other downstream targets that mediate the tumor-suppressive role of miR-873.

We used samples from CRC patients and two CRC mouse models to test the diagnostic and prognostic values of miR-873. Although our results showed that low miR873 level may be related to CRC progression, further investigations that employ a much larger-scale approach are required to determine whether it could serve as a reliable biomarker for clinical detection. Collectively, our study highlights the novel roles of miR-873 in alleviating malignant proliferation and metastasis of CRC cells.

\section{MATERIALS AND METHODS}

\section{Clinical specimens}

55 paired CRC samples and their normal counterparts were acquired from the Department of Surgical Oncology, First Affiliated Hospital of Xiamen University (Xiamen, China). Tissues were immediately put into Liquid Nitrogen after surgery and stored at $-80^{\circ} \mathrm{C}$ until RNA isolation. Experiments were performed in according to the guidelines by the Ethics and Scientific Committees of Xiamen University with consent of the patients.

\section{Cell culture}

CRC cell lines SW620 and HCT116 were kindly provided by Dr. Han You (School of Life Sciences, Xiamen University). HCT8, SW480, LS174T, HT29 and RKO were obtained from the Institute of Biochemistry and Cell Biology, Chinese Academy of Sciences, Shanghai. 293 T cells were obtained from ATCC. HCT116 cells were maintained in McCoy's 5A supplemented with $10 \%$ fetal bovine serum (FBS). 293T, SW620, LS174t and HT29 cells were cultured in DMEM supplemented with $10 \%$ FBS. HCT8, SW480, RKO and LoVo cells were maintained in RPMI-1640 media supplemented with $10 \%$ FBS.

\section{Animal studies}

$A P C^{M i n+}$ mice were kindly provided by Dr. Jiahuai Han (School of Life Sciences, Xiamen University). The colitis-associated CRC mouse model was constructed as previously described [9]. For in vivo cell growth assays, $5 \times 10^{6}$ cells were subcutaneously injected into the lower back region of 6-8 weeks-old male nude mice for 28 days ( $n=5$ per group). For experimental liver metastasis model, $5 \times 10^{5}$ cells were intrasplenically injected into the 6-8 weeks-old male nude mice for 28 days ( $n=5$ per group). Bioluminescence imaging was performed in the live animal Lumina II system (Xenogen IVIS system).

\section{Plasmid construction, cell transfection and infection}

Plasmid construction was done as previously described [32]. The Has-pre-miR-873 flank region was inserted into pCDH-CMV-EF1 $\alpha$-puro vector and 3'UTR regions containing the predicted miR-873 binding sites were amplified and inserted into the pMIR-REPORT Vector. Primers used for plasmid construction were listed in Supplementary Table 1. For generation of stable cell lines, a lentivirus-based packaging system (pMDL, REV 
and VSVG) was used [33, 34]. MiR-873 inhibitor and the negative control inhibitor were designed and synthesized by Genepharma (Shanghai, China). For cell transfection, Lipofectamine 2000 (Invitrogen) was used according to the manufacturer's instruction. In vitro experiments were performed and total RNA and protein were isolated 48 hours after transfection.

\section{Cell proliferation assays}

For MTT assay, $2 \times 10^{3}$ cells were seeded in 96well plates. MTT was added to the medium and the absorbance at $490 \mathrm{~nm}$ was tested every day until the $5^{\text {th }}$ day. For colony formation assay, 300 cells were seeded in 6-well plates. After two weeks, cells were fixed, stained and photographed. Experiments were performed in triple.

\section{Cell migration and invasion assays}

Transwell plates (Corning) containing $8 \mu \mathrm{m}$ pore size membranes were used for migration (without matrigel) or invasion (with matrigel) assays. For migration assays, $5 \times 10^{4}$ LoVo, HCT116 cells or HCT8 cells were seeded in the top chamber. For invasion assays, $1 \times 10^{5}$ LoVo, HCT116 cells or HCT8 cells were seeded in the top chamber. After 48 hours, cells on the lower flat were fixed, stained and photographed. Experiments were performed at least for three times.

\section{Dual Luciferase reporter assays}

MiR-873-overexpressing plasmid or vector and 3'UTR-Luciferase plasmids were co-transfected into $293 \mathrm{~T}$ cells seeded in a 24 -well plate. Cell were lysed 48 hours after transfection and activities of Luciferase and Rellina were measured by the Dual-Glo Luciferase Assay System (Promega). Experiments were performed for three times.

\section{Quantitative real-time PCR (qRT-PCR)}

Cell or tissues were lysed by the Trizol reagent (Roche) and total RNA was isolated. TaqMan MicroRNA Reverse Transcription Kit (ABI) was used for miRNA reverse transcription. ReverTra Ace qPCR RT Kit (Toyobo) was applied for mRNA reverse transcription. TransStart Top Green qPCR SuperMix (TransGen Biotech) was used for quantification of miRNA/mRNA level. GAPDH (for mRNA) and U6 (for miRNA) were used respectively as positive control and the $2^{-\Delta \Delta C t}$ algorithm was applied for relative quantification of mRNA and miRNA levels. Primers used for qRT-PCR were listed in Supplementary Table 2.

\section{Western blotting}

Western blotting was performed as previously described [32]. Immunoblot was carried using the following primary antibodies: ELK1 and STRN4 (Abcam); Vimentin (R\&D); CyclinD1 (Santa Cruz); E-Cadherin and N-Cadherin (BD); $\alpha$-E-catenin, ERK1/2 and p-ERK (Cell Signaling); $\beta$-actin (Thermo Fisher).

\section{Abbreviations}

3'UTR, 3'-untranslated-region; AOM/DSS, azoxymethane/dextran sodium sulfate; CDK6, cyclin dependent kinase 6; ELK1, ETS transcription factor; MyoB1, myosin IB; STRN4, striatin 4; TRAF2, TNF receptor associated factor 2; WASF2, WAS protein family member 2; NSCLC, non-small cell lung cancer; MTT, 3-(4,5-dimethyl-2-thiazolyl)-2,5-di-phenyl-2-Htetrazolium bromide; FBS, fetal bovine serum.

\section{Author contributions}

C.F., B.L., D.C. and M.Z. performed the experiments. C.F. and Z.M. performed bioinformatics analyses. Z.H. and Y.Z. prepared tumor specimen and mRNA. F.L. provided technical support. C.F., D.C. and Y.L. analyzed the data and wrote the paper.

\section{ACKNOWLEDGMENTS AND FUNDING}

This study was supported by grants from the National Natural Science Funds of China (No. 81471970), the Natural Science Foundation of Fujian Province of China (No. 2015J01349), the Open Research Fund of State Key Laboratory of Cellular Stress Biology, Xiamen University (No. SKLCSB2017KF007), the Science and Technology Project of Natural Science Foundation of Fujian Province (No. 2016J01639), the Medical Innovations Topic in Fujian Province (No. 2016-CXB-8).

\section{CONFLICTS OF INTEREST}

Chuannan Fan, Biyu Lin, Zhengjie Huang, Dan Cui, Minyi Zhu, Zhenling Ma, Yunda Zhang, Fan Liu and Yingfu Liu declare that they have no conflict of interest.

\section{REFERENCES}

1. Jemal A, Bray F, Center MM, Ferlay J, Ward E, Forman D. Global cancer statistics. CA Cancer J Clin. 2011; 61:69-90. https://doi.org/10.3322/caac.20107. [PubMed]

2. Siegel RL, Miller KD, Fedewa SA, Ahnen DJ, Meester RG, Barzi A, Jemal A. Colorectal cancer statistics, 2017. CA Cancer J Clin. 2017; 67:177-193. https://doi.org/10.3322/ caac.21395. [PubMed]

3. Sjöblom T, Jones S, Wood LD, Parsons DW, Lin J, Barber TD, Mandelker D, Leary RJ, Ptak J, Silliman N. The consensus coding sequences of human breast and colorectal cancers. Science. 2006; 314:268-74. https://doi. org/10.1126/science.1133427. [PubMed] 
4. Network CGA. Comprehensive molecular characterization of human colon and rectal cancer. Nature. 2012; 487:330-7. https://doi.org/10.1038/nature11252. [ubMed]

5. Dienstmann R, Vermeulen L, Guinney J, Kopetz S, Tejpar $\mathrm{S}$, Tabernero J. Consensus molecular subtypes and the evolution of precision medicine in colorectal cancer. Nat Rev Cancer. 2017; 17:79-92. https://doi.org/10.1038/ nrc.2016.126. [PubMed]

6. Jin K, Li T, van Dam H, Zhou F, Zhang L. Molecular insights into tumour metastasis: tracing the dominant events. J Pathol. 2017; 241:567-577. https://doi.org/10.1002/ path.4871. [PubMed]

7. Vilar E, Tabernero J. Molecular dissection of microsatellite instable colorectal cancer. Cancer Discov. 2013; 3:502-11. https://doi.org/10.1158/2159-8290.CD-12-0471. [PubMed]

8. Matano M, Date S, Shimokawa M, Takano A, Fujii M, Ohta Y, Watanabe T, Kanai T, Sato T. Modeling colorectal cancer using CRISPR-Cas9-mediated engineering of human intestinal organoids. Nat Med. 2015; 21:256-62. https://doi. org/10.1038/nm.3802. [PubMed]

9. Fan C, Lin Y, Mao Y, Huang Z, Liu AY, Ma H, Yu D, Maitikabili A, Xiao H, Zhang C, Liu F, Luo Q, Ouyang G. MicroRNA-543 suppresses colorectal cancer growth and metastasis by targeting KRAS, MTA1 and HMGA2. Oncotarget. 2016; 7:21825-39. https://doi.org/10.18632/ oncotarget.7989. [ [PubMed]

10. Vennin C, Spruyt N, Dahmani F, Julien S, Bertucci F, Finetti P, Chassat T, Bourette RP, Le Bourhis X, Adriaenssens E. H19 non coding RNA-derived miR-675 enhances tumorigenesis and metastasis of breast cancer cells by downregulating c-Cbl and Cbl-b. Oncotarget. 2015; 6:29209-23. https://doi.org/10.18632/oncotarget.4976. [PubMed]

11. Wang RJ, Li JW, Bao BH, Wu HC, Du ZH, Su JL, Zhang MH, Liang HQ. MicroRNA-873 (miRNA-873) inhibits glioblastoma tumorigenesis and metastasis by suppressing the expression of IGF2BP1. J Biol Chem. 2015; 290:893848. https://doi.org/10.1074/jbc.M114.624700. [PubMed]

12. Wu D, Li X, Meng X, Yan J, Zong Z. MicroRNA-873 mediates multidrug resistance in ovarian cancer cells by targeting ABCB1. Tumour Biol. 2016; 37:10499-506. https://doi.org/10.1007/s13277-016-4944-y. [PubMed]

13. Cui J, Yang Y, Li H, Leng Y, Qian K, Huang Q, Zhang C, Lu Z, Chen J, Sun T. MiR-873 regulates ER $\alpha$ transcriptional activity and tamoxifen resistance via targeting CDK3 in breast cancer cells. Oncogene. 2015; 34:3895-907. https:// doi.org/10.1038/onc.2014.430. [PubMed]

14. Gao Y, Xue Q, Wang D, Du M, Zhang Y, Gao S. miR873 induces lung adenocarcinoma cell proliferation and migration by targeting SRCIN1. Am J Transl Res. 2015; 7:2519-26. eCollection 2015. [PubMed]

15. Suzuki H, Takatsuka S, Akashi H, Yamamoto E, Nojima M, Maruyama R, Kai M, Yamano HO, Sasaki Y, Tokino T. Genome-wide profiling of chromatin signatures reveals epigenetic regulation of MicroRNA genes in colorectal cancer. Cancer Res. 2011; 71:5646-58. https://doi. org/10.1158/0008-5472.CAN-11-1076. [PubMed]

16. Moser A, Luongo C, Gould KA, McNeley M, Shoemaker A, Dove W. ApcMin: a mouse model for intestinal and mammary tumorigenesis. Eur J Cancer. 1995; 31A:1061-4. [PubMed]

17. Grivennikov S, Karin E, Terzic J, Mucida D, Yu G-Y, Vallabhapurapu S, Scheller J, Rose-John S, Cheroutre H, Eckmann L. IL-6 and Stat3 are required for survival of intestinal epithelial cells and development of colitisassociated cancer. Cancer cell. 2009; 15:103-13. https:// doi.org/10.1016/j.ccr.2009.01.001. [PubMed]

18. Neufert C, Becker C, Türeci Ö, Waldner MJ, Backert I, Floh K, Atreya I, Leppkes M, Jefremow A, Vieth M. Tumor fibroblast-derived epiregulin promotes growth of colitis-associated neoplasms through ERK. J Clin Invest. 2013; 123:1428-43. https://doi.org/10.1172/JCI63748. [PubMed]

19. Hatley ME, Patrick DM, Garcia MR, Richardson JA, Bassel-Duby R, Van Rooij E, Olson EN. Modulation of K-Ras-dependent lung tumorigenesis by MicroRNA-21. Cancer Cell. 2010; 18:282-93. https://doi.org/10.1016/j. ccr.2010.08.013. [PubMed]

20. Thiery JP, Acloque H, Huang RY, Nieto MA. Epithelialmesenchymal transitions in development and disease. Cell. 2009; 139:871-90. https://doi.org/10.1016/j. cell.2009.11.007. [PubMed]

21. Sonoshita M, Itatani Y, Kakizaki F, Sakimura K, Terashima T, Katsuyama Y, Sakai Y, Taketo MM. Promotion of colorectal cancer invasion and metastasis through activation of NOTCH-DAB1-ABL-RHOGEF protein TRIO. Cancer Discov. 2015; 5:198-211. https://doi.org/10.1158/21598290.CD-14-0595. [PubMed]

22. Shenouda SK, Alahari SK. MicroRNA function in cancer: oncogene or a tumor suppressor? Cancer Metastasis Rev. 2009; 28:369-78. https://doi.org/10.1007/s10555-009-91885. [PubMed]

23. Lin S, Gregory RI. MicroRNA biogenesis pathways in cancer. Nat Rev Cancer. 2015; 15:321-33. https://doi. org $/ 10.1038 / \mathrm{nrc3932}$. [PubMed]

24. Bu P, Wang L, Chen KY, Rakhilin N, Sun J, Closa A, Tung KL, King S, Varanko AK, Xu Y. miR-1269 promotes metastasis and forms a positive feedback loop with TGF- $\beta$. Nat Commun. 2015; 6:6879. https://doi.org/10.1038/ ncomms7879. [PubMed]

25. Chen X, Zhang Y, Shi Y, Lian H, Tu H, Han S, Peng B, Liu W, He X. miR-873 acts as a novel sensitizer of glioma cells to cisplatin by targeting Bcl-2. Int J Oncol. 2015; 47:160311. https://doi.org/10.3892/ijo.2015.3143. [PubMed]

26. Chai Y, Chipitsyna G, Cui J, Liao B, Liu S, Aysola K, Yezdani M, Reddy ESP, Rao VN. c-Fos oncogene regulator Elk-1 interacts with BRCA1 splice variants BRCA1a/1b and enhances BRCA1a/1b-mediated growth suppression in breast cancer cells. Oncogene. 2001; 20:1357-67. https:// doi.org/10.1038/sj.onc.1204256. [PubMed] 
27. Cohen-Armon M, Visochek L, Rozensal D, Kalal A, Geistrikh I, Klein R, Bendetz-Nezer S, Yao Z, Seger R. DNA-independent PARP-1 activation by phosphorylated ERK2 increases Elk1 activity: a link to histone acetylation. Mol Cell. 2007; 25:297-308. https://doi.org/10.1016/j. molcel.2006.12.012. [PubMed]

28. Ohta H, Aoyagi K, Fukaya M, Danjoh I, Ohta A, Isohata N, Saeki N, Taniguchi H, Sakamoto H, Shimoda T. Cross talk between hedgehog and epithelial-mesenchymal transition pathways in gastric pit cells and in diffuse-type gastric cancers. Br J Cancer. 2009; 100:389-98. https://doi. org/10.1038/sj.bjc.6604846. [PubMed]

29. Duan Q, Pang C, Chang N, Zhang J, Liu W. Overexpression of PAD4 suppresses drug resistance of NSCLC cell lines to gefitinib through inhibiting Elk1-mediated epithelialmesenchymal transition. Oncol Rep. 2016; 36:551-8. https://doi.org/10.3892/or.2016.4780. [PubMed]

30. Hyodo T, Ito S, Hasegawa H, Asano E, Maeda M, Urano T, Takahashi M, Hamaguchi M, Senga T. Misshapenlike kinase 1 (MINK1) is a novel component of striatininteracting phosphatase and kinase (STRIPAK) and is required for the completion of cytokinesis. J Biol Chem. 2012; 287:25019-29. https://doi.org/10.1074/jbc. M112.372342. [PubMed]
31. Wong M, Hyodo T, Asano E, Funasaka K, Miyahara R, Hirooka Y, Goto H, Hamaguchi M, Senga T. Silencing of STRN4 suppresses the malignant characteristics of cancer cells. Cancer Sci. 2014; 105:1526-32. https://doi. org/10.1111/cas.12541. [ubMed]

32. Lin Y, Liu AY, Fan C, Zheng H, Li Y, Zhang C, Wu S, Yu D, Huang Z, Liu F, Luo Q, Yang CJ, Ouyang G. MicroRNA33b Inhibits Breast Cancer Metastasis by Targeting HMGA2, SALL4 and Twist1. Sci Rep. 2015; 5: 9995. https://doi.org/10.1038/srep09995. [PubMed]

33. Fang X, Cai Y, Liu J, Wang Z, Wu Q, Zhang Z, Yang C, Yuan L, Ouyang G. Twist2 contributes to breast cancer progression by promoting an epithelial-mesenchymal transition and cancer stem-like cell self-renewal. Oncogene. 2011; 30:4707-20. https://doi.org/10.1038/onc.2011.181. [PubMed]

34. Liu AY, Cai Y, Mao Y, Lin Y, Zheng H, Wu T, Huang Y, Fang $X$, Lin S, Feng Q. Twist2 promotes self-renewal of liver cancer stem-like cells by regulating CD24. Carcinogenesis. 2014; 35:537-45. https://doi.org/10.1093/ carcin/bgt364. [PubMed] 